\title{
Individual genomes and personalized medicine: life diversity and complexity
}

\section{"...insights into genetic variation and molecular mechanisms underlying human diversity have improved our understanding of the complexity of life and disease heterogeneity."}

Patients and tumors are unique. The conceptual design is great. Identifying the genetic variants underlying phenotype can lead to personalized medicine. Tailoring the best medical intervention to the right individual or patient can dramatically improve health. A decade after the first draft of human genome sequence $[1,2]$ and the promises of a health revolution, what progress has been made in both genomics and personalized medical practice and what are the challenges and perspectives to deal with for the next decade?

The use of personalized medicine to improve both the prevention and cure of disease is potentially achievable through: predicting both the disease risk among healthly individuals in the general population and the therapeutic response among patients. Genomic information from individuals or patients can substantially contribute to biomarker-based guided personalized prevention and treatment.

The first strategy to use personalized medicine, in the prevention setting, involves identifying high-risk individuals that may develop major common diseases, such as cardiovascular disorder, diabetes and cancer, and then selecting the most appropriate preventive intervention to protect them from these diseases. This strategy can substantially reduce disease incidence and it is particularly important for hard-to-treat disorders, such as cancer. However, despite current research efforts, the development of robust biomarkers based on primary prevention has only modestly improved.

In the second strategy, in the treatment setting, efforts by academia and industry have been focused on how to improve diagnostics and prognosis of diseases and how, through the development of predictors of drug response and adverse effects, to improve the safety and efficacy of drugs. Indeed, not only is the therapeutic response rate for several complex diseases low, but general toxicity rates of currently used agents are also still alarmingly high.
Several research strategies and scientific fields have been developed towards personalized medicine. Pharmacogenetics, studying the genetic associations with drug efficacy and toxicity has led to the identification of several dugmetabolizing enzymes, with the most important belonging to the cytochrome P450 family [3]. The aim is to predict adverse effects to guide individualized treatment. Despite overlap with pharmacogenetics, the term pharmacogenomics is distinct because it evaluates the application of genomics to drug discovery. Pharmacogenomics involves the mechanism of the action of drugs on cells as revealed by gene-expression patterns. Pharmacoproteomics provides a more functional representation of patient-to-patient variation than that which is provided by genotyping, and therfore also contributes to personalized medicine. A 'pharmacometabonomic' approach involves the study of metabolites and how these can contribute to personalizing drug treatment.

\section{Human genome}

In June 2000, at a gala televised press conference attended by the then US President Bill Clinton and UK Prime Minister Tony Blair, Francis Collins (NIH, MD, USA), the leader of the international publicly funded Human Genome Project [1], and Craig Venter (J Craig Venter Institute, MD, USA), the leader of the private forprofit company Celera Genomics (CA, USA) [2], jointly announced the completion of their first draft of human genome sequence. The scenario of the race between two rivals from the public and private sector was built up perfectly by the media. The White House press statement articulated the hope, felt by many, that this landmark achievement would "lead to a new era of molecular medicine, an era that will bring new ways to prevent, diagnose, treat and cure disease" [4].

Now, at the end of the first post-genome decade, where will we stand and where will we be 10 years from now? Taking lessons from the

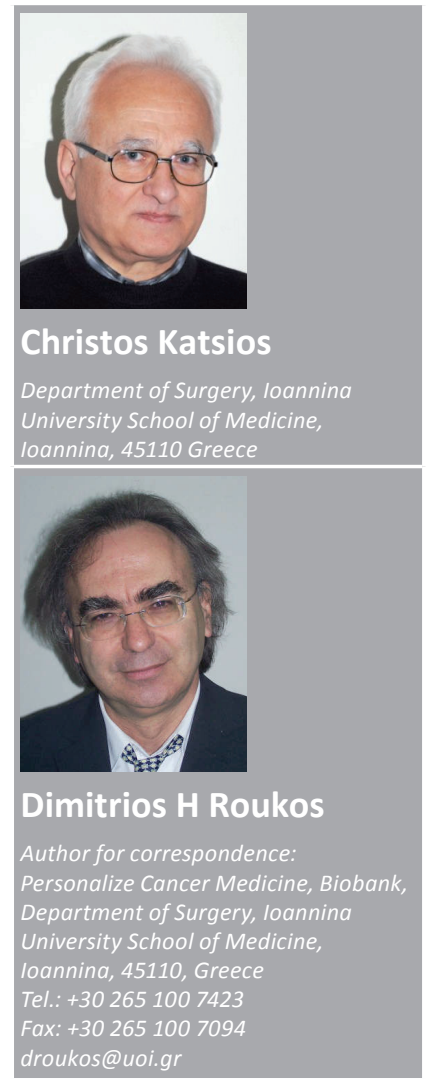

future medicine $^{\text {pits }}$ fs 
long history of science with only a few basic science discoveries being translated into medicine, what has been the progress in both genomics and healthcare?

\section{Genomics revolution}

The first post-genome decade was characterized by spectacular advances in genome science. The dramatic improvement in DNA sequencing technology with a drop of costs by approximately 14,000-fold has increased the base pairs of sequence in databases from 8 billion in 2000 to 270 billion bases today, in 2010 [5]. Two dozen human genomes have been completely sequences and published, while approximately another 200 have been sequenced but not published [4]. Many other 'big biology' efforts, such as the International HapMap Project, and the Encyclopedia of DNA Elements (ENCODE), provide important information on gene-coding and noncoding DNA with the aim of improving our understanding of every functional element in the human genome. Much noncoding DNA has a regulatory role; small RNAs of different varieties seem to control gene expression at the level of both DNA and RNA transcripts in ways that are still only beginning to become clear [6].

Based on sequence databases, microchips with tens of thousands of genetic variants, including SNPs and copy number variants, have been developed. Over the last 3 years these chips have allowed genome-wide association studies (GWAS) to identify of a wealth of novel genetic variants associated with diseases. Soon, biotechnology companies, including Celera Genomics, deCODE Genetics in Reykjavik, Iceland, will have to rethink their optimistic assumption that selling human genetic information could turn into a huge profit. Why do these genome-wide advances provide little clinical application?

\section{Life complexity \& biodiversity}

The gap between basic research and clinical application has been widened. The more we learn, the bigger the problem becomes of understanding the mysteries of biodiversity, aging and complex diseases, such as cancer [6]. Yet, the implications of the genomic revolution in medicine and health are limited. To move forward to the future Francis Collins considers five key lessons: personalized medicine, technology, policy, partnerships and pharmacogenomics [7]. Craig Venter emphasizes the need for research on linking genotype to phenotype and points out that, because of the myriad phenotypic traits as clinical data, more powerful computational strategies will be needed to link phenotype to genotype [8].

Indeed, there are several reasons explaining the limitations in translating genomic discoveries into medical practice. Major hurdles, which will have to be overcome in order to achieve personalized medicine for improving health in the future can be summarized into the completion of somatic mutations catalogue, understanding human and disease variability, and predicting complex gene-environment interactions.

Why has medical practice not moved forward in parallel with the genomics explosion? First, recent genome-wide studies using massively parallel DNA sequencing technology have revealed, for example, for breast cancer, that not only point mutations, such as nucleotide insertions, deletions and SNPs, but also genomic rearrangements and copy number changes are involved in tumorigenesis for breast, lung and other major cancers [9-14]. Second, these studies have demonstrated a widespread variability of all these somatic mutations [15-17], so that thousands of human genomes and cancer genomes should be completely sequenced to have a complete landscape of causal mutations for each individual cancer patient. Third, there is the bigger challenge of understanding how the complex gene-gene, protein-protein, gene-environment and cancer cell-cell interactions, in a timely and very dynamic process, are involved in the transformation of normal cells to disease cells, such as cancer cells, at a certain time point [18-21].

\section{"...the implications of the genomic revolution in medicine and health are limited."}

Summarizing the results of GWAS towards personalized medicine, two categories of genetic variants have emerged. The first group includes very rare mutations present in the general population that increase the risk of diseases such as schizophrenia, epilepsy or autism by up to 20 -fold. These discoveries have clinical applications in risks prediction but, because of their rarity, the benefit is limited to only a few only individuals, The second group includes common genetic variants but they confer only a very small increase in disease risk, and therefore, have little or no clinical utility [22]. Indeed, only nine and ten SNPs identified by GWAS that may be involved in cardiovascular disease and breast cancer, respectively, did not seem to improve disease-risk prediction [23,24]. 
The challenge: genotype-phenotype map prediction

Both genotype and phenotype are crucial for disease-risk prediction among individuals $[7,8]$. As the costs drop and quality of sequencing data is improved, the catalog of driver mutations for major diseases will be improved. High-quality clinical and therapeutic data (phenotype) are available from large-scale randomized controlled trials and databases. The bigger challenge now is how to link all these phenotype data to the genotyping data.

\section{“...human genomes and cancer genomes that have been completely sequenced, and the revolution in genomics have together revealed the high complexity and heterogeneity of cancer and other common complex diseases."}

Yet the nonlinear relationship between genotype and phenotype represents the biggest challenge in biomedical and mathematical sciences. Several computational strategies are being developed to predict gene-gene and gene-environment interactions [21]. Bionetworks modeling represents one of the most promising research fields towards a genotype-phenotype-based personalized medicine [25]. Efforts are underway to integrate genotyping and molecular data into molecular network modeling to predict such outcomes [20]. Systems biology, oncology and medicine open up new ways to understand complex biological systems and how it could be possible to link genomic data with clinical data and disease. This would lead the way to understanding the pathogenesis of disease and biomarkers for disease risk prediction and preventions could be developed [19,20,22,26-28].

\section{Conclusion}

At the end of the first post-genomic era with its explosion in sequencing technology, important advances and insights into genetic variation and molecular mechanisms underlying human diversity have improved our understanding of the complexity of life and disease heterogeneity. However, the dramatic increase in the number of human genomes and cancer genomes that have been completely sequenced, and the revolution in genomics have together revealed the high complexity and heterogeneity of cancer and other common complex diseases. This complexity of an unpreceded level explains the little impact, at least for now, that genomics has in a day-to-day clinical practice. This suggests the need for an international network of scientific collaboration and conceptual innovation to overcome a myriad of problems, and the labyrinth of life and disease complexity to achieve personalized medicine [29-34].

\section{Financial \& competing interests disclosure}

The authors have no relevant affiliations or financial involvement with any organization or entity with a financial interest in or financial conflict with the subject matter or materials discussed in the manuscript. This includes employment, consultancies, honoraria, stock ownership or options, expert testimony, grants or patents received or pending, or royalties.

No writing assistance was utilized in the production of this manuscript.

\section{Bibliography}

1 Lander ES, Linton LM, Birren B et al.; International Human Genome Sequencing Consortium: Initial sequencing and analysis of the human genome. Nature 409, 860-921 (2001).

2 Venter JC, Adams MD, Myers EW et al: The sequence of the human genome. Science 291, 1304-1351 (2001).

3 Roederer MW: Cytochrome P450 enzymes and genotype-guided drug therapy. Curr. Opin. Mol. Ther. 11(6), 632-640 (2009).

4 The human genome at ten. Nature 464(7289), 649-650 (2010).

5 Check Hayden E: Human genome at ten: life is complicated. Nature 464(7289), 664-667 (2010).

6 Human genome at ten: the sequence explosion. Nature 464(7289), 670-671 (2010).
Collins F: Has the revolution arrived? Nature 464(7289), 674-675 (2010).

8 Venter JC: Multiple personal genomes await. Nature 464(7289), 676-677 (2010).

9 Stratton MR, Campbell PJ, Futreal PA: The cancer genome. Nature 458(7239), 719-724 (2009).

10 Pleasance ED, Stephens PJ, O'Meara S et al:: A small-cell lung cancer genome with complex signatures of tobacco exposure. Nature 463(7278), 184-190 (2010).

11 Pleasance ED, Cheetham RK, Stephens PJ et al.: A comprehensive catalogue of somatic mutations from a human cancer genome. Nature 463(7278), 191-196 (2010).

12 Shah SP, Morin RD, Khattra J et al.: Mutational evolution in a lobular breast tumour profiled at single nucleotide resolution. Nature 461(7265), 809-813 (2009).
13 Stephens PJ, McBride DJ, Lin ML et al.: Complex landscapes of somatic rearrangement in human breast cancer genomes. Nature 462(7276), 1005-1010 (2009).

14 Beroukhim R, Mermel CH, Porter D et al: The landscape of somatic copy-number alteration across human cancers. Nature 463(7283), 899-905 (2010).

15 Heppner GH: Tumor heterogeneity. Cancer Res. 44(6), 2259-2265 (1984).

$16 \mathrm{He}$ Y, Wu J, Dressman DC, IacobuzioDonahue $\mathrm{C}$ et al: : Heteroplasmic mitochondrial DNA mutations in normal and tumour cells. Nature 464(7288), 610-614 (2010).

17 Jones S, Zhang X, Parsons DW: Core signaling pathways in human pancreatic cancers revealed by global genomic analyses. Science 321(5897), 1801-1806 (2008).

18 Hanahan D, Weinberg RA: The hallmarks of cancer. Cell 100, 57-70 (2000). 
19 Roukos DH: Systems medicine: a real approach for future personalized oncology?

Pharmacogenomics 11(3), 283-287 (2010).

20 Schadt EE: Molecular networks as sensors and drivers of common human diseases. Nature 461(7261), 218-223 (2009).

21 Rockman MV: Reverse engineering the genotype-phenotype map with natural genetic variation. Nature 456, 738-744 (2008).

22 Goldstein DB: 2020 visions: personalized medicine. Nature 463(7277), 26-32 (2010).

23 Kathiresan S, Melander O, Anevski D et al.: Polymorphisms associated with cholesterol and risk of cardiovascular events. $N$. Engl. J. Med. 358(12), 1240-1249 (2008).

24 Wacholder S, Hartge P, Prentice R et al: Performance of common genetic variants in breast-cancer risk models. N. Engl. J. Med. 362(11), 986-993 (2010).

25 Roukos DH: Bionetworks-based personalized medicine versus comparative-effectiveness research or harmonization of both in cancer management? Expert Rev. Mol. Diagn. 10(3), 247-250 (2010).

26 Weinberg R: Point: hypotheses first. Nature 464(7289), 678 (2010).

27 Roukos DH, Ziogas D: From tumor size and HER2 status to systems oncology for very early breast cancer treatment. Expert Rev. Anticancer Ther. 10(2), 123-128 (2010).

28 Roukos DH: Novel clinico-genome network modeling for revolutionizing genotype-phenotype-based personalized cancer care. Expert Rev. Mol. Diagn. 10 (1), 33-48 (2010).

29 Ziogas D, Roukos DH: Genetics and personal genomics for personalized breast cancer surgery: progress and challenges in research and clinical practice. Ann. Surg. Oncol. 16(7), 1771-1782 (2009).

30 Roukos DH: Personalized cancer diagnostics and therapeutics. Expert Rev. Mol. Diagn. 9(3), 227-229 (2009).
31 Roukos DH: Radiation therapy for breast cancer. N. Engl. J. Med. 360(13), 1362; author reply 1363 (2009).

32 Roukos DH: Targeting gastric cancer with trastuzumab: new clinical practice and innovative developments to overcome resistance. Ann. Surg. Oncol. 17, 14-17 (2010).

33 Roukos DH: Twenty-One-Gene Assay: challenges and promises in translating personal genomics and whole-genome scans into personalized treatment of breast cancer. J. Clin. Oncol. 27(8), 1337-1338 (2009).

34 Roukos DH: Personal genomics and genome-wide association studies: novel discoveries but limitations for practical personalized medicine. Ann. Surg. Oncol. 16(3), 772-773 (2009). 